of pyorrhcea alveolaris in adult life is largely determined by the supply of vitamin A which was available during the period of growth. These few illustrations must suffice to indicate that the original conception of vitamin-deficiency diseases has extended its boundaries to include a variety of diseases attributable to past dietary deficiencies, preventable by suitable feeding during the period of growth.

Similar results have been achieved during the period under review by the study of specific mineral deficiency diseases. Prominent among those mineral elements deficiency of which in the diet may lead to recognisable symptoms of disease are iron, copper, iodine, phosphorus, calcium and magnesium. Lack of sufficient iron in the food has been shown to result frequently in the development of anæmia. Such 'nutritional' anæmia is particularly common in infants, and is accounted for by the fact that milk is a poor source of iron. The recognition of this form of anæmia has proved to be of considerable practical importance, because it is often associated with an increased susceptibility to many of the common complaints of infancy and can be corrected with great ease. The enormous amount of work that has lately been carried out on the relation between iodine deficiency and the development of goitre has not yet completely solved the problem of the causes of thyroid enlargement, but it has certainly provided very successful methods of wholesale prophylaxis in districts where goitre is endemic. Lastly may be mentioned the revolution in the cattle-rearing industry of South Africa which has followed the discovery of phosphorus deficiency as the immediate or ultimate cause of serious losses in this branch of agriculture.

There can be no question that already the modern conception of nutrition has produced practical results of immense significance in both preventive and curative clinical and veterinary medicine. The results are not confined to the prophylaxis and treatment of diseases which arise as the result of actual deficiencies in the food supplied. They have already been extended to include the treatment of diseases in which defects in the absorption or utilisation of particular food elements rather than faulty diets are responsible for the development of co-existing signs of deficiency diseases. The term 'secondary deficiency disease' is now being used to distinguish this particular class of nutritional disorder. In this group might well be placed that once fatal malady pernicious anæmia, which the brilliant researches of the past decade have shown to be amenable to simple dietetic treatment, although there is no evidence that dietetic errors play any part in its causation. As more and more precise information is gained concerning the intimate processes of metabolism within the body, there should be increasing opportunities of preventing and successfully treating disease by adjusting the diet to influence those metabolic processes which may produce the symptoms of disease when they deviate from their normal course. This has long been one of the aims of clinical medicine; its ultimate realisation has surely been brought one stage nearer fulfilment by the nutritional investigations of the past twenty-five years.

\title{
Viruses as the Cause of Disease
}

By Dr. Joseph A. Arkwright, F.r.s., Lister Institute of Preventive Medicine, London

$\mathrm{A}^{\mathrm{T}}$ the end of the nineteenth century, a new category of infective agents was discovered which are now classed as viruses, in the modern sense of the word. The chief property which unites them, and by which they have been distinguished from previously known minute parasites, is the extremely small size of their component particles, since these are smaller than bacteria, and many of them are not visible even with the highest powers of the microscope. Until quite recently, it was customary to speak of all viruses as invisible, but in some cases the minute granules of which the virus appears to consist can be clearly seen, when stained, by direct microscopic observation; but in most cases they cannot be distinguished by their shape, but only by their uniformity, numbers and their source in special parts of the diseased tissues. The recent investigations by
Barnard with the ultra-microscope and photomicrography by ultra-violet light have added to our knowledge of their size and form.

A special feature by which the invasion of the cells of the host by many viruses can be recognised is the occurrence of 'cell inclusion bodies'. These forms, of which there may be one or more in a single cell, vary in size and may be larger than the nucleus. Opinion as to their nature has undergono various vicissitudes. After their discovery, when they were at first hailed as protozoal parasites, they were for long regarded as merely reaction products of the cell protoplasm to the presence of the virus, a position now favoured for the 'inclusion bodies' associated with virus diseases of plants. More recently it has been shown that in some virus diseases of animals these inclusion bodies' consist of masses of the minute filterable 
forms of 'elementary bodies' held together by a soluble matrix. These bodies have been most completely and fruitfully studied in fowl-pox, small-pox, vaccinia, ectromelia-a disease of mice -and psittacosis, the infective disease of parakeets which also attacks man.

The list of diseases of man and animals due to filterable viruses is continually being increased, and considerably more than fifty are now known; their study has been intensified and especially productive during the last fifteen years.

The original recognition of the existence and importance of these agents was due to the use of earthenware and porcelain filters which retained the smallest bacteria. When tissue extracts or secretions from an infected animal were passed through such filters, the filtrates were shown to be infective and capable of reproducing the disease in a fresh animal; this process could be repeated indefinitely, proving that the active agent multiplied in the animal body and was not merely a chemical substance or toxin. The virus of footand-mouth disease, the first shown to cause a disease of animals, was discovered in 1898 by Loeffler and Frosch. It passes through finer filters than any other known virus, so that no question of visible particles has arisen, since massed granules or inclusion bodies have not been observed. The most essential qualifying characteristic of a virus has ever since been its filterability.

Most viruses remain active after being dried over sulphuric acid, and some are more resistant to alcohol and certain other disinfectants than bacteria. Many viruses are present in the tissue juices in high concentration, and such suspensions in liquids can still prove infective when diluted 1 in $10^{5}$ or 1 in $10^{6}$. The resemblance in many respects of a virus to an excessively minute bacterium has led to the belief in their similar nature which is now held by most pathologists. It must be admitted, however, that part of the argument is based on analogy, since viruses cannot be subjected to the same tests as bacteria to prove that they are living agents causing disease.

Besides the small size of the ultimate particles of a virus and the resulting absence of a recognisable differentiating morphology, there are certain other peculiarities distinguishing these two classes of agents. Bacteria, with few cxceptions, can be propagated on sterilised artificial culture media, and can be obtained in pure culture by the method introduced by Koch of selecting single colonies grown on a solid sterile medium. By this means their infective and other activities can be examined without the risk of contamination with substances derived from the host. A virus, on the other hand, in most cases requires the presence of living cells of the host to enable it to multiply, and it often appears to grow only or chiefly inside the cells of the animal tissues. Artificial culture of a virus can, however, very often be maintained in pieces of animal tissue kept alive and growing apart from the body. The virus of fowl-pox, vaccinia or vesicular stomatitis of the horse and of some other diseases can also be propagated in the living embryo in an incubated hen's egg.

The fact that tissues of a host are needed to enable viruses to multiply has led to the suggestion that the virus may not necessarily be alive but may only serve as a stimulus to the host cells, causing them to reproduce the virus, and that the particles seen in a suspension containing a virus and indeed the virus itself are really products of the host.

This suspicion has been especially strong in the case of the infective transmissible sarcoma of fowls described by Rous, which can be reproduced by injecting a filtered cell-free extract of the diseased tissues into a normal fowl. The resemblance of avian sarcoma to other virus diseases extends to the recognition by Ledingham and Gye, by the use of the high-speed centrifuge, of minute particles resembling those of other viruses. The nature of these particles was moreover confirmed by their reaction (agglutination) with the blood serum of animals which had been injected with the sarcoma, in the same way that similar reactions have been demonstrated with the elementary bodies from other virus diseases.

The resemblance in structure and behaviour of the sarcoma of fowls to the malignant growths of mammals gives rise to hesitation before admitting that it is caused by an extrinsic virus, since mammalian malignant tumours have never been found to yield an infective cell-free extract and have been usually regarded as due to intrinsic tissue changes, though both mammalian and avian sarcomata can be induced by external physical and chemical irritants, such as tar and certain other substances.

If therefore an extrinsic virus is one of the essential causes of fowl sarcoma, it must already be present in every susceptible fowl. The view that viruses which produce disease are not essentially invading parasites, but are produced by the host, is opposed by the regularity with which diverse viruses can be propagated in the same kind of animal, and by the fact that the same virus may infect several widely different species. For example, there are three distinct types of foot-andmouth disease virus which produce apparently identical symptoms in animals and can only be distinguished by the fact that any one does not protect an animal against infection with the other two; nevertheless, each virus maintains its identity whether propagated in the cow, pig, 
guinea pig, rat or hedgehog. It is difficult to see how this could happen if the virus were produced by each species of mammal from its own tissues.

Another filterable agent, in many respects resembling the virus of an animal disease, is the bacteriophage, which was independently discovered by Twort and d'Herelle. The effect of a drop of a suspension of bacteriophage added to a young liquid culture of susceptible bacteria is that the latter are dissolved and a large amount of fresh phage is produced. The bacterium-free filtrate of the liquid culture may often be diluted ten million times and still the same effect be produced by a drop as by the original suspension.

D'Herelle and many other bacteriologists believe that the phage is a living agent which infects young growing bacteria, multiplies in their interior, and is set free when the bacteria die and break up.

Phage, though destroyed at a temperature of $70^{\circ}-75^{\circ} \mathrm{C}$., as a rule survives at $60^{\circ}-65^{\circ} \mathrm{C}$., when the bacteria with which it is associated are killed; it also resists drying and is remarkably resistant to the action of alcohol, acetone and chloroform. D'Herelle considers that all strains of phage are really one, though different strains become adapted to different bacteria, but more probably many phages when first obtained are a mixture of distinct races, and most filtrates containing phage are in the first instance derived from sewage or fæces, containing a great variety of bacteria.

Some strains of bacteria harbour a phage although apparently insusceptible to its destructive action. The activity is only manifested when a filtrate is tested on another susceptible strain. Thus many bacterial cultures have been shown to produce phage, though the presence of phage is not apparent and may not even have been suspected. This phenomenon suggests the original production of the phage by an uninfected culture, but it may merely be another instance of an apparently normal organism 'carrying' a parasite ; many parallel cases are known of animals and plants 'carrying' infective agents whilst themselves unaffected. De Jong showed that cultures of certain sporing bacilli which produce a phage may still be 'lysogenic' after being heated at $100^{\circ} \mathrm{C}$. for five minutes, whereas the free phage is killed at $70^{\circ} \mathrm{C}$. for five minutes. When the spores germinate the phage is again liberated. This experiment suggests that the phage is preserved by its inclusion in the resistant spore, and this evidence of its derivation from the germinating spore de novo is not conclusive.

The chief reason for doubting the living nature of some phages and certain viruses is the very small size of their filtrable particles, which makes it very doubtful whether they can have a complex composition resembling that of other living things.
Different strains of phage are very unequal in their filterability; some have relatively large particles with a diameter about half those of vaccinia, while others pass through very fine filters, like the virus of foot-and-mouth disease, for which the diameter is estimated at about one tenth of the coarser phages.

The uniform and carefully graded collodion membranes introduced by Elford, of which the average pore diameter can be calculated, enables much closer estimates to be made than formerly of the size of particles which just pass or are just withheld. The size of the particles of some viruses has also been calculated, especially by Bechhold, by their rate of deposition when centrifuged at 10,000-15,000 rev. per min.

It has been possible to purify virus particles by first filtering and then centrifuging at high speed, washing the deposit and again centrifuging, as has been shown by Ledingham.

By the new collodion ultra-filters the diameter of the particles of different viruses has been estimated to vary from $200 \mathrm{~m} \mu$ to $150 \mathrm{~m} \mu$ for vaccinia to about 8-10 $\mathrm{m} \mu$ for foot-and-mouth disease $(\mu=$ mikron $=1$ thousandth of a millimetre; $m \mu=$ one thousandth of a mikron). It is difficult to understand how with such dimensions they can have a composition of sufficient complexity to consist of living matter. For comparison, the smallest bacteria have a diameter of $1 \cdot 0-0 \cdot 5 \mu$ and the egg-albumen molecule has been estimated at $4 \cdot 34 \mathrm{mu}$ diameter.

Doerr, in a recent treatise, while granting that some viruses have been shown to be living, denies the possibility of life in those of the smaller dimensions. It so happens that the viruses of foot-and-mouth disease and louping-ill, which are among those with the smallest particles, exhibit all the typical essential characters of viruses both in vitro and in the animal body, though causing very different diseases and having very different 'life-histories'.

The exact and quantitative experiments with filters have been made possible by the high concentration in which certain viruses occur, and by opportunities for deternaining the presence of the virus in different dilutions by inoculation of susceptible small animals. The dilution of some fluids containing virus from the animal body can be carried to 1 in $10^{8}$ or even higher when dealing with foot-and-mouth disease, vaccinia and some other diseases without depriving them of infectivity.

The quandary arising from the very active and apparently vital functions of virus particles in spite of their small size raises the question whether the accepted definitions of life are universally applicable or whether some intermediate state 
between what is called living and dead matter may not exist, as has been suggested by Boycott.

Of the functions usually postulated for a living organism, assimilation appears to be the most characteristic and indispensable. It is reasonable to assume that the metabolism of an organism would be much simplified if it existed in a circulating medium which provided a constantly changing supply of materials resembling its own components, such as might be afforded for an obligatory parasite living inside the cells of its host. Such an existence would have very different requirements from a truly independent life.

Virus diseases are transmitted from one animal to another by very varied means. Some, like canine distemper and certain influenza-like diseases of man, by droplets in the breath, others like yellow and dengue fevers by the bites of insects, others like louping-ill of sheep by the bites of blood-sucking ticks or of mites; again, the bite of the mammalian host is the usual mode of infection with rabies, but for many others the method of transmission is still uncertain. In these respects they do not differ from diseases due to bacteria.

It is characteristic of many diseases that, although the initial infection is caused by a virus, many of the symptoms and complications are due to secondary infections with bacteria, and this is notably the case in the influenza-like group in man, in swine fever, canine distemper and swine influenza.

A virus may become remarkably adapted and sometimes permanently attenuated, when transferred to a new host, as is well instanced in the change of the virus of small-pox to vaccinia in cattle and rabbits, and of the rabies virus in the rabbit.

The period of resistance shown by the host following an attack of disease is sometimes very prolonged, even lifelong, after small-pox, varicella, yellow fever and canine distemper, but in some other cases the protection afforded is of comparatively short duration, in foot-and-mouth disease usually for one to two years, whereas frequently recurring attacks due to the virus of Herpes labialis are common.
This immunity is to a great extent due to the production in the animal body of 'antibodies' which can be found in the blood serum of recovered animals, just as occurs after bacterial infection. These antibodies can often be demonstrated by the formation of a precipitate or by the agglutination of the virus particles when a suspension of the elementary bodies is mixed with the serum, or by the neutralisation of the virus by the serum when both are injected into an animal. These phenomena are of the same kind as the precipita tion occurring when the blood serum of an animal which has been inoculated with a foreign protein (antigen) is mixed with the same protein in vitro, and are not peculiar to true infections.

It is not intended here to do more than refer to the enormous and increasing number of filterable viruses known to cause infective disease in plants and found in their juices. These, like mosaic disease of tobacco, spotted wilt of tomato, and crinkle and leaf-roll of potato, may cause very serious disease, or in other cases may be present throughout the plant without producing any visible effect, as in some infections of the potato.

There is good evidence that two viruses may co-exist in the same plant, and as a result the symptoms may be either much more or much less severe than when either virus is present alone.

Some viruses are transmitted by insects such as aphis or thrips, while others pass by unknown means. In some of these diseases of plants peculiar 'inclusion bodies' are found in certain cells, but their relation to the virus is undetermined. It is known that these 'bodies', as well as some of the symptoms due to a virus, can in special cases be imitated by the addition of certain inorganic salts to the soil, but the disease is not then transmissible. Some plant viruses are highly resistant to drying, chemical action and alcohol, and in many ways the viruses of plants resemble those of animals.

The problems which the behaviour and properties of viruses raise are of great practical and theoretical interest, and are by no means yet solved.

\section{Heat Production of Muscle and Nerve}

\section{By Prof. A. V. HiLl, o.B.E., F.R.s., Foulerton Research Professor of the Royal Society}

$\mathrm{T}^{\mathrm{T}}$ HE first paper by the present writer on this subject was printed almost exactly twentyfive years ago in the Journal of Physiology: the coincidence reinforced the invitation of the Editor of NATURE to write an interim report.

The heat production of muscle had been investigated in the past by such scientific giants as
Helmholtz, Heidenhain, Fick and Blix: and indeed it was with Blix's apparatus, purchased by the providence of Langley and set in order by the Cambridge Instrument Company in the days of Horace Darwin and Keith Lucas, that the present experiments began. For some time, apart from Blix's work on the relation between heat 